
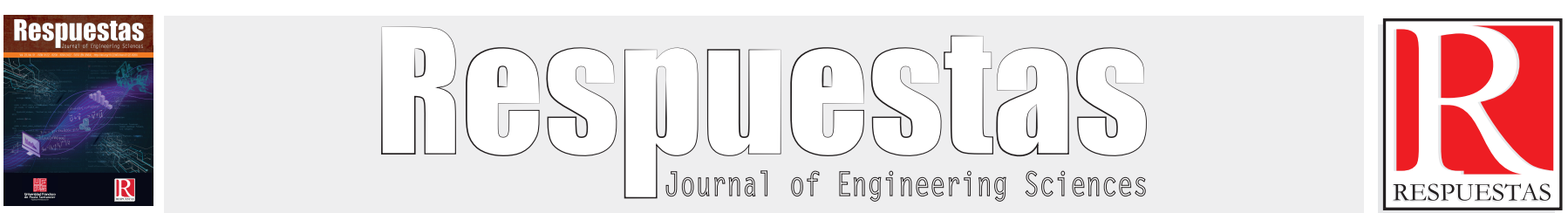

Artículo Original

https://doi.org/10.22463/0122820X.1499

\title{
El número, como instrumento de medición y formación de una realidad materica, abstracta y matemática
}

The number, as an instrument of measurement and formation of a materic, abstract and mathematical reality

Juan Manuel Villa-Carrero, Julián Orlando-Tarazona.

${ }^{a}$ Master en Teória e Historia, juanmanuelvc@ufps.edu.co, orcid.org/0000-0003-4978-4611, Universidad Francisco de Paula Santander, Cúcuta, Colombia.

${ }^{b}$ Magister en Ingeniería Electrónica, juliantarazona@ufps.edu.co, orcid.org/0000-0003-0777-786X, Universidad Francisco de Paula Santander, Cúcuta, Colombia.

Cómo citar: J.M. Villa-Carrero y J. Orlando-Tarazona, "El número, como instrumento de medición y formación de una realidad materica, abstracta y matemática", Respuestas, vol. 23, no. S1, pp. 38 - 41, 2018. https://doi.org/10.22463/0122820X.1499

Recibido: Febrero 01, 2018; Aprobado: Julio 10, 2018.

\begin{tabular}{ll}
\hline & RESUMEN \\
\hline Palabras Clave: & $\begin{array}{l}\text { El presente trabajo consiste en trascender lo perceptual, ese primer conocimiento de alguna cosa a través de los sentidos, para } \\
\text { optar por el número, como instrumento de medición y formación de una realidad materica, abstracta y matemática en el diseño } \\
\text { de nuevos objetos arquitectónicos. En concreto, la problemática en este trabajo fue elaborar herramientas electrónicas que } \\
\text { permitan extraer información fuera del alcance de la realidad experiencial del diseñador y explorar métodos de modelado de las } \\
\text { Diseño Realidad } \\
\text { Materialismo dialéctico } \\
\text { dinámicas de la realidad. Como resultado, este trabajo permitió, a través de sensores de sonido, medir y dar forma a estas } \\
\text { habituales en los análisis arquitectónicos. Lo anterior favoreció el control y significación de los diseños de nuevos objetos. Lo } \\
\text { cual, es fundamental, si comprendemos que los objetos fabricados por el hombre desnaturalizan el mundo para acercarlo más a } \\
\text { objetivos humanos y/o artificiales. Todo esto parece confirmar, la falta de un mayor compromiso por parte de los diseñadores } \\
\text { con lo real, más allá de un mundo de símbolos, en la era del antropoceno. }\end{array}$ \\
\hline ABSTRACT \\
\hline Keywords: & $\begin{array}{l}\text { The present work consists of transcending the perceptual, that first knowledge of something through the senses, to opt for } \\
\text { number, as an instrument of measurement and formation of a material, abstract and mathematical reality in the design of new } \\
\text { architectural objects.The problem in this work was to develop electronic tools that allow extracting information from the reach } \\
\text { of the experiential reality of the designer and explore methods of modeling the dynamics of reality. As a result, this work } \\
\text { allowed, through sound sensors, to measure and shape these dynamics and process them digitally through spatio-temporal maps, } \\
\text { instead of the usual conventional maps usual in architectural analysis. This favored the control and significance of the designs } \\
\text { Dialectical Materialism } \\
\text { of new objects. }\end{array}$ \\
\hline
\end{tabular}

\section{Introducción}

Este proyecto de investigación surgió al interior de la práctica de diseño del programa de Arquitectura de la Universidad Francisco de Paula Santander- UFPS, en la cual, pusimos en duda el compromiso por parte de los diseñadores con la realidad. Esta cuestión viene siendo asumida en los últimos años en diferentes proyectos de diseñadores que ven en los datos que arroja la realidad una herramienta de diseño. De manera que, dentro de una línea de pensamiento pitagórica iniciamos esta investigación, en la cual, un componente cuantitativo como el número puede explicar el mundo y no la apariencia sensible [1].

Así que, esta investigación en general tuvo como objetivo comprender lo real, entendido como una "realidad objetiva", que de acuerdo a Slavoj Zizek [2],[3] se está convirtiendo cada vez más en una estructura formal abstracta que se basa en el trabajo científico y experimental complejo. De modo que, en este trabajo la realidad (empíricamente real) se enfrentará a lo Real (modelo abstracto o mapeos matemáticos, etc.).

En particular, junto con los cursos integradores del programa de Electricidad y Electrónica de la UFPS decidimos unir esfuerzos en una estrategia pedagógica de investigación aplicada que ayudará a través de la ciencia y tecnología a comprender lo real. Fig. 1 Por lo cual, juntos desarrollamos un artefacto como un medio entre la realidad, en la cual, los humanos confrontan cosas, y su humanidad. En otras palabras, esta investigación buscó comprender los contornos de la realidad a través del número, el cual, faculta al hombre, no solo a conocer lo real fuera del alcance sensorial, sino que también permite dar forma [4].

\section{Materiales y métodos}

En consecuencia, para transformar el conocimiento teórico en conocimiento práctico y útil, que permitiera utilizar el número como instrumento de formación en procesos de diseño, el grupo multidisciplinario formalizó una metodología que se abrevia en tres pasos: Primero, la reflexión e implementación de métodos alternativos de observación e interpretación de la temática teórica descrita, al interior de la práctica pedagógica.[5],[6] En segundo lugar, diseño y fabricación del artefacto en mención, y por último, la validación y verificación del

*Autor de correspondencia.

E-mail: juanmanuelvc@ufps.edu.co (Juan Manuel Villa Carrero),

(c) (i) (3) $\odot$ Peer review is the responsibility of the Universidad Francisco de Paula Santander. (c) 1 Gy $\mathrm{ND}$ This is an article under the license CC BY-NC-ND 
producto y sus resultados.

El primer paso, consistió en implementar estrategias pedagógicas dentro de la práctica de diseño que pusiera en contacto a los estudiantes con métodos alternativos al tradicional, y aparatos analógicos y digitales para observar e interpretar la realidad. Estos métodos, permitieron, capturar y modelar las dinámicas de la naturaleza, a partir de mapas espacio temporales. Los cuales, llevaron al dicente hacia un pensamiento de carácter científico, inclinado a la objetividad, separado de prejuicios y representaciones preconcebidas, las cuales, dificultan el diseño.

El segundo paso, incluyo a estudiantes de los cursos integradores del programa de electricidad y electrónica. Los cuales, aplicaron e integraron teorías de circuitos, programación y manejo de sensores, propios de su disciplina, a partir de los requerimientos de forma, función y propósito, formulados para este ejercicio pedagógico desde el micro currículo de la práctica de diseño de arquitectura.

En este orden de ideas, y con el uso de la tecnología libre Arduino,[7],[8] tanto estudiantes de electrónica, como de arquitectura fabricaron dos artefactos para entender la realidad a través del sonido, o lo que podríamos denominar un SONAR. Cuya función en este caso fue enfrentar a lo Real (modelo abstracto o mapeos matemáticos, etc.), con la realidad (empíricamente real), y/o la función de medir en tiempo real las cosas en la realidad empírica a través de sensores de ultrasonido, tanto a objetos estáticos en el espacio tridimensional, como en movimiento. Estos artefactos, capturan datos y los procesan a través de una tarjeta de Arduino [9],[10],[11] la cual, conecta su puerto serial con una hoja de cálculo en Excel, que permite exportar los datos a diversos software para dar forma a mapeos espacio temporales, o permiten trasladar lo real y enfrentarlo a nuestra realidad experiencial.
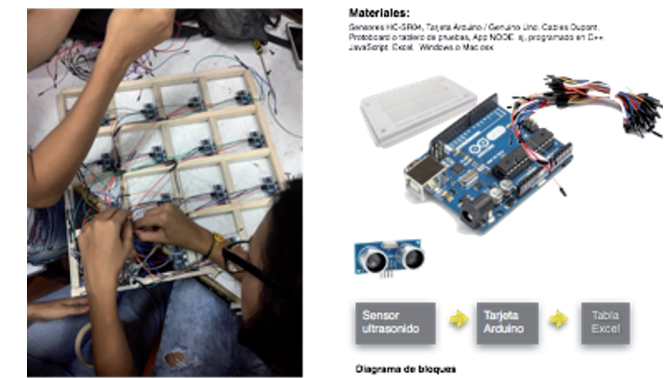

Figura 1. Estudiantes de electrónica y arquitectura fabricando el artefacto en forma de grilla con hardware libre Arduino

El primer artefacto fabricado, en forma de una vara, debió deslizarse de acuerdo al tiempo que pasa entre el envío y la recepción del ultrasonido,[12],[13] para que de ese modo pudiera lograr la captura de datos de una realidad tridimensional estática. Así mismo, esta forma lineal facilito ordenar los sensores y rastrillar la realidad, hasta configurar una rejilla tridimensional de datos, que percibe e interpreta una realidad tridimensional, cuyo volumen de captura es: eje $X=1 \mathrm{~m}$, por eje $Y=\infty$, y eje $Z=2 m$. Fig. 2

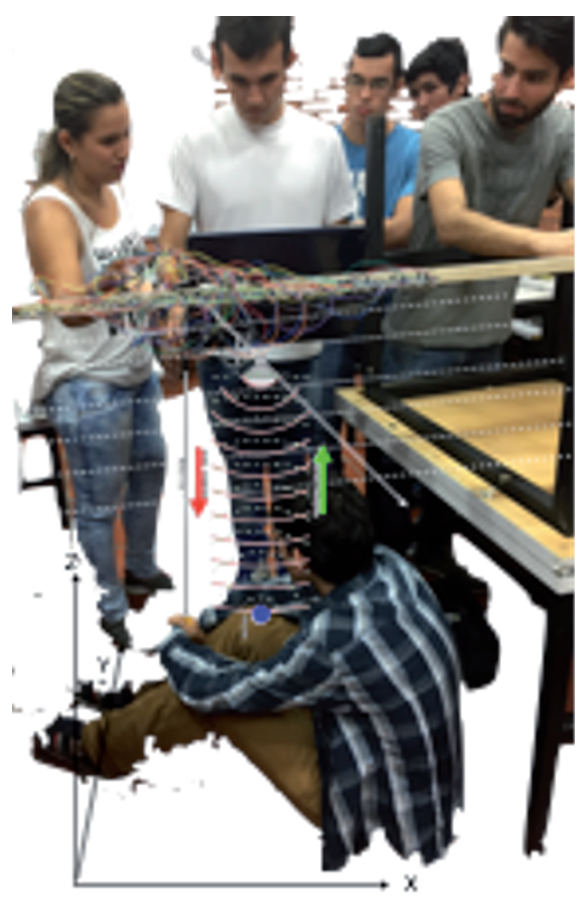

Figura 2. Captura de datos prueba 1

El segundo artefacto fabricado, en forma de una retícula, debió permanecer estática, para conseguir capturar el espacio-tiempo tácito bajo la grilla. La forma de esta retícula ordeno la red de sensores que capturan el espacio

tridimensional en relación con el tiempo que trascurre entre el envío y la recepción del ultrasonido, hecho que logra percibir e explicar una realidad tridimensional en movimiento, cuadro a cuadro. Cuyo volumen de captura es: eje $\mathrm{X}=0.60 \mathrm{~m}$, eje $\mathrm{Y}=$ $0,60 \mathrm{~m}$, eje $\mathrm{Z}=2 \mathrm{~m}$ y un eje de tiempo $\mathrm{T}=\infty$. Fig. 3

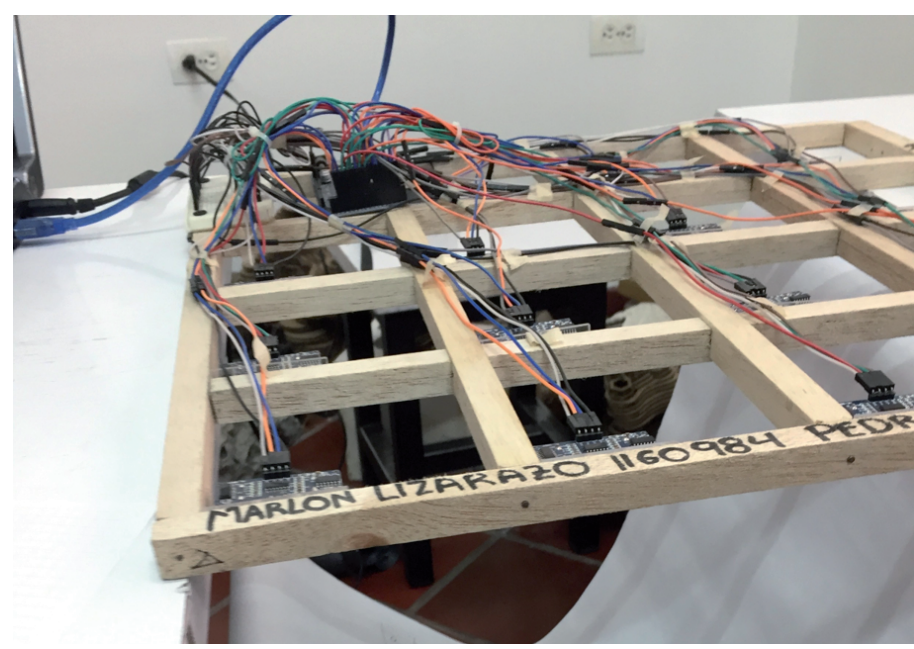

Figura 3. Artefacto 2 


\section{Resultados y análisis}

El último paso verificó y validó los resultados. Es decir, logramos a través del número enfrentar la realidad empírica con una realidad matérica, abstracta y matemática. En particular, efectuamos tres pruebas:

La primera prueba uso el primer artefacto para capturar datos de un cuerpo humano inmóvil. Y la segunda prueba uso el artefacto en forma de grilla para capturar datos de agua en movimiento. Los resultados de la captura de los datos de las dos primeras pruebas fueron interpretados por el grupo de estudiantes de arquitectura, en relación a la estructura de orden del artefacto, y a la lógica funcional o artificio de cada prueba. La tercera prueba fue realizada fuera de este proceso pedagógico en los laboratorios de simulación del departamento de arquitectura SIMU-lab. Esta última prueba involucro software digitales de simulación, como Rhino,[14] y Grasshopper, [15] directamente conectados al software de los artefactos fabricados, como Node y Excel.

Así pues, la primera hoja de cálculo resultado de la primera prueba fue interpretada en dos formas: la primera relacionó los valores obtenidos de las mediciones del espacio en diferentes tonalidades de color hasta crear una imagen del objeto observado. La segunda, relacionó los valores obtenidos en un software de modelado 3d,[16] el cual, simuló el objeto medido. Fig. 4, 5

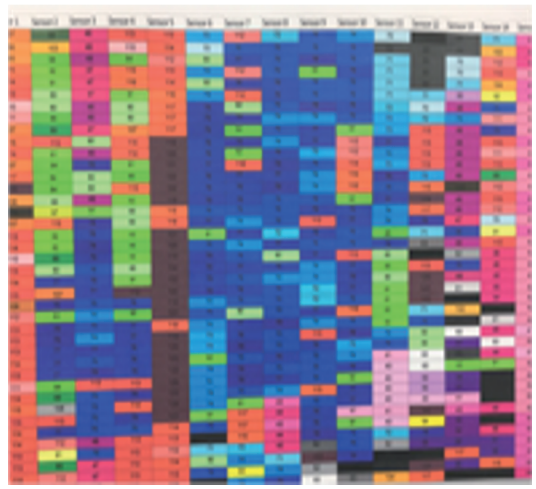

Figura 4. Hoja de cálculo, resultado de la captura de datos cuerpo estático en la primera prueba

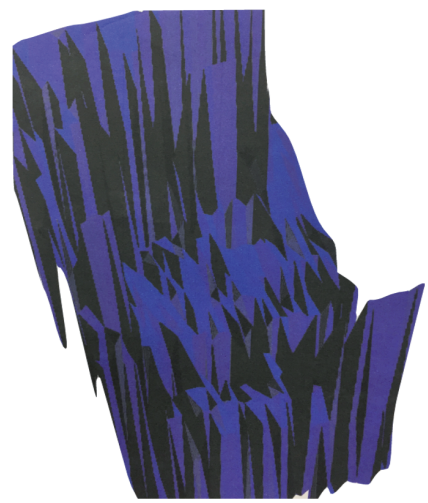

Figura 5. Hoja de cálculo, resultado de la captura de datos cuerpo estático en la primera prueba
La segunda prueba arrojo datos del espacio 3d, cuadro a cuadro, a intervalos de tiempo predeterminados por el sensor de sonido. Es decir, midió las variaciones de la superficie del agua, y genero una capa por cada segundo rastreado. Estos datos fueron interpretados en una animación en $3 \mathrm{~d}$ hasta crear una simulación del agua en movimiento. Fig. 6

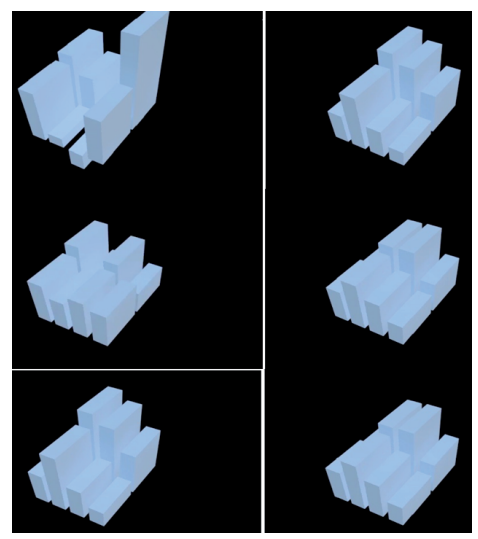

Figura 6. Animación resultado captura de datos artefacto 2, cuerpo de agua en movimiento

La tercera y última prueba, al igual que la segunda, usaron la retícula de sensores, es decir arrojó resultados de objetos en movimiento. La diferencia consistió en que la interpretación de datos de esta última prueba no dependió de factores humanos como las dos pruebas anteriores. En esta prueba, en cambio los registros obtenidos del movimiento de un pliego de papel, fueron procesados directamente de una hoja de cálculo de Excel a una fórmula del plugin Grasshoper, de Rhinos. Esta fórmula, simuló el espacio y tiempo de captura de los datos, y así pudo reproducir los movimientos o dinámicas de lo real a través de mapas y modelosgeométricos producto de funciones matemáticas. Fig. 7, 8.

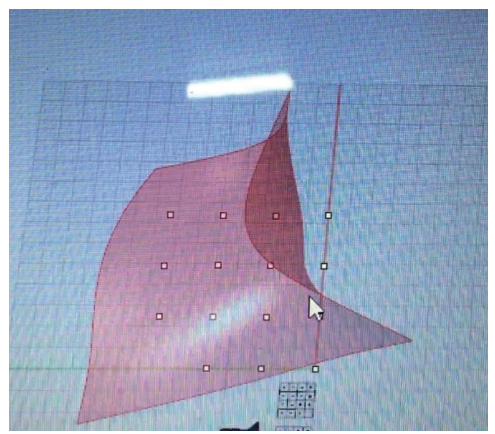

Figura 7. Visualización de datos del objeto (hoja de papel) en Grasshoper, de Rhinos

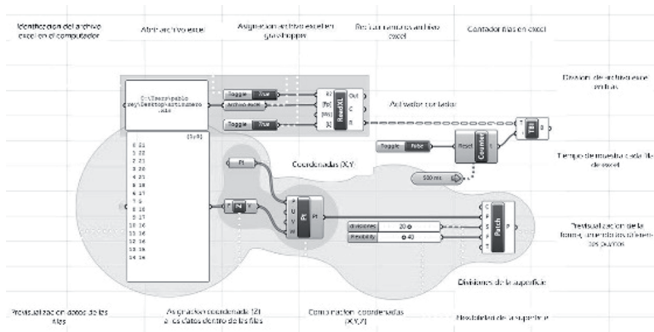

Figura 8. Definición de Grasshoper para visualizar en Rhinos 


\section{Conclusiones}

En conclusión, el número da forma y permite comprender lo Real, y confrontarlo con nuestra realidad empírica. Así mismo, dispositivos que median entre la realidad y nuestra humanidad, o que nos permiten penetrar lo real y/o comprenderlo, no solo nos conecta con el entendimiento de lo Real, sino que además, nos permite construir nuevos objetos dentro de un mayor control. Estos objetos desnaturalizan el mundo para acercarlo más a objetivos humanos y/o artificiales. Esto evidencia la necesidad de un mayor compromiso por parte de los diseñadores con lo real, más allá del mundo sensible, en la era del antropoceno.

\section{Colaboradores y agradecimientos}

March Javier Lemus, así como, Estudiantes de Arquitectura, Taller V segundo cuatrimestre 2016, estudiante de arquitectura y sistemas Pablo Rey Taller IV, II, 2017, y estudiantes de Electrónica, Marlon Lizarazo, Pedro Beltrán y María Laguado de la asignatura de cursos integradores, Universidad Francisco, por último, agradezco al $\mathrm{PhD}(\mathrm{c})$ Ing. Ricardo Bermúdez por su desinteresada colaboración para que este proyecto interdisciplinar pudiera realizarse.

\section{Referencias}

[1] A. Takahashi, «El hechizo de Pitagoras el discreto encanto de la geometria,» Ideas Valores, vol. 55, nº 131, pp. 97-111, 2006.

[2] S. Zizek, "Absolute Recoil Towards A New Foundation Of Dialectical Materialism", http://www.english.ufl.edu, 2014. [Online]. Available: http://www.english.ufl.edu/mrg/readings/Zizek,\%20Absolute\%20Recoil\%20introduction.pdf. [Accessed: 12- Sep- 2016].

[3] S. Zizek, Slavoj Zizek, Vida examinada (Examined life, Astra Taylor, 2008).

[4] A. G. Tyng, Interviewee, "Number is Form and Form is Number". [Entrevista]. spring 2005.

[5] L. Halprin, The RSVP Cycles: Creative Processes in the Human Environment, New York: Paperback, 1970.

[6] E. Miralles y E. Prats, «"How to Lay out a Croissant”,» El Croquis, $n^{\circ} 49-50,1991$.

[7] "Arduino - Home", Arduino.cc, 2018. [Online]. Available: https://www.arduino.cc/. [Accessed: 19- Oct- 2016].

[8] "Arduino - Tutorials", Arduino.cc. [Online]. Available:
https://www.arduino.cc/en/Tutorial/ HomePage. [Accessed: 19- Oct- 2016].

[9] Mario de La Horra Kollmer, Sistemas de adquisición de datos basados en la plataforma arduino. Aplicaciones a matlab, simulink y android. Universidad Carlos III de Madrid, Leganés, España, Mayo. 2013.

[10] A. López Orozco and J. Aricapa Quiceno, Implementación de un sistema de adquisición de datos para el análisis cinemático en un mecanismo plano. Colombia: Universidad Tecnológica de Pereira. Facultad de Tecnologías. Tecnología Mecánica, 2015.

[11] Joaquín Cruz Isabel, Sistema de adquisición de datos mediante waspmode, Universidad Carlos III de Madrid, Leganés, España, Abril. 2012.

[12] Martínez Fuentes, "Introducción a la plataforma Arduino y al Sensor ultrasónico HC-SR04", Profesional, Universidad Carlos III de Madrid, 2014.

[13] Freaks, Elec. Ultrasonic Ranging Module hc-sr04. HC-SR04 datasheet (2016). [Online]. Available: https://cdn.sparkfun.com/datasheets/Sensors/Proximity/HCSR04.pdf

[14] "Rhino 6 para Windows", Rhino3d.com, 2018 .[Online]. Available:https:/www.rhino3d. com/es/. [Accessed: 19- Oct2016].

[15] "Tutorials", Grasshopper3d.com. [Online]. Available:https://www.grasshopper3d.com/page/tutorials-1. [Accessed: 12- Oct- 2016].

[16] "Free Software for Students \& Educators | 3ds Max | Autodesk", Autodesk.com. [Online]. Available:https://www.autodesk.com/education/ free-software/3ds-max. [Accessed: 05Oct- 20] 